\section{Case Reports in Ophthalmology}

\title{
Demyelinizing Neurological Disease after Treatment with Tumor Necrosis Factor- $\alpha$ Antagonists
}

\author{
Claudia Bruè Cesare Mariotti Ilaria Rossiello Andrea Saitta \\ Alfonso Giovannini \\ Ophthalmology, Department of Neuroscience, Polytechnic University of Marche, \\ Ancona, Italy
}

\section{Keywords}

Tumor necrosis factor antagonists - Optic neuritis · Adalimumab

\begin{abstract}
Purpose: Demyelinizing neurological disease is a rare complication after treatment with tumor necrosis factor (TNF) $\alpha$ antagonists. We report on a case of multiple sclerosis after TNF $\alpha$ antagonist treatment and discuss its differential diagnosis. Methods: This is an observational case study. Results: A 48-year-old male was referred to Ophthalmology in January 2015 for an absolute scotoma in the superior quadrant of the visual field in his right eye. Visual acuity was 20/50 in the right eye and 20/20 in the left. Fundus examination was unremarkable bilaterally. Spectral domain optical coherence tomography revealed a normal macular retina structure. Visual field examination revealed a superior hemianopsia in the right eye. Head magnetic resonance imaging showed findings compatible with optic neuritis. The visual evoked potentials confirmed the presence of optic neuritis. The patient had been under therapy with adalimumab since January 2014, for Crohn's disease. Suspension of adalimumab was recommended, and it was substituted with tapered deltacortene, from $1 \mathrm{mg} / \mathrm{kg} / \mathrm{day}$. After 1 month, the scotoma was resolved completely. Conclusions: TNF $\alpha$ antagonists can provide benefit to patients with inflammatory autoimmune diseases. However, they can also be associated with severe adverse effects. Therefore, adequate attention should be paid to neurological abnormalities in patients treated with TNF $\alpha$ antagonists.
\end{abstract}




\section{Case Reports in Ophthalmology}

\section{Introduction}

Tumor necrosis factor (TNF) $\alpha$ antagonists have been widely used to treat autoimmune diseases. They are an effective treatment; however, there is the risk of severe adverse effects, which can include demyelinating disorders. TNF has toxic effects on oligodendrocytes in vitro, and anti-TNF agents were effective in preventing autoimmune encephalomyelitis in a mouse model of autoimmune demyelination. However, in rare cases, trials on anti-TNF agents have revealed adverse events that have included immune activation and aggravation of disease [1-4]. Furthermore, the temporal proximity of anti-TNF agents, the occurrence of multiple sclerosis, and the recovery from these symptoms after discontinuation of these agents strongly suggest a direct association between multiple sclerosis and anti-TNF agents. The demyelinating disorders that have been reported to be connected with TNF antagonists are variable and have included multiple sclerosis, optic neuritis, transverse myelitis, and Guillain-Barré syndrome.

The mechanisms underlying the predisposition to demyelination or exacerbation of demyelination in patients treated with $\mathrm{TNF} \alpha$ antagonists is not well understood. Several hypotheses have been proposed [4]. TNF $\alpha$ has a critical role in multiple sclerosis. It is clearly a pro-inflammatory cytokine during the acute phase of multiple sclerosis and participates in the demyelinating process. On the other hand, TNF $\alpha$ also has immunosuppressive properties during the later phase of multiple sclerosis. These properties are related to TNF receptor (TNFR) 1 and TNFR2, which mediate the differential biological responses of TNF $\alpha$. A review of the Adverse Events Reporting System database of the Food and Drug Administration in 2001 identified 20 cases of demyelinating disorders following treatment with anti-TNF $\alpha$ agents for arthritis [2]. Optic neuritis was described as the second most common demyelinating disorder (8 of 20), and it was the sole presenting symptom in two of the cases [2].

We report here on the clinical findings for spectral domain optical coherence tomography (SD-OCT), and magnetic resonance imaging (MRI) of a man affected by Crohn's disease, who had been treated with adalimumab for 1 year. To the best of our knowledge, this is the first case that has used oral prednisolone to treat optic neuritis after the use of adalimumab in a patient with Crohn's disease.

\section{Case}

A 48-year-old male was referred to Ophthalmology in January 2015 for an absolute scotoma in the superior quadrant of the visual field in his right eye. Visual acuity was 20/50 in the right eye and 20/20 in the left. Fundus examination was unremarkable bilaterally (fig. 1a). SD-OCT revealed a normal macular retina structure (fig. 1b). However, visual field examination identified a superior hemianopsia in the right eye (fig. 2). Head MRI showed findings of optic neuritis. His visual evoked potentials (VEPs) were reduced, with prolonged latency, which was compatible with an optic neuritis diagnosis (fig. 3).

The patient was affected by Crohn's disease, which had failed to respond to methotrexate, sulfasalazine, leflunomide and oral prednisolone, both alone and in combination, and at their full doses. He had then been on adalimumab 40 mg since January 2014, a year previously. We recommended suspension of his adalimumab use, and it was substituted with the synthetic anti-inflammatory glucocorticoid deltacortene $1 \mathrm{mg} / \mathrm{kg} /$ day. This dosing was tapered to terminate within 3 weeks. 
One month from the withdrawal of the adalimumab treatment and initiation of this prednisolone therapy, computerized perimetry showed complete resolution of his previous visual field defects in the affected eye. At that time, his VEP testing also showed improved responses, although the latency in the affected eye remained longer than in the other eye. No further deterioration or relapse was noted during the follow-up, and his VEP and visual field investigations at 12 months were entirely normal.

\section{Discussion}

Adalimumab is a recombinant monoclonal antibody that binds to the cytokine TNF $\alpha$ [57]. TNF $\alpha$ antagonists have been found to be efficacious in the treatment of many immunemediated inflammatory diseases, including rheumatoid arthritis, polyarticular juvenile rheumatoid arthritis, Crohn's disease, psoriatic arthritis, and ankylosing spondylitis.

$\mathrm{TNF} \alpha$ is a pro-inflammatory cytokine during the acute phase of multiple sclerosis, and it participates in the demyelinating process. Then, during the later phase of multiple sclerosis, TNF $\alpha$ shows immunosuppressive properties. These properties are mediated through TNFR1 and TNFR2, which are responsible for the differential biological responses of TNF $\alpha$. Within the central nervous system, TNF $\alpha$ is mainly generated by microglia and astrocytes, as the monomeric transmembrane precursor protein (tmTNF). The cytoplasmic tail is then cleaved by the TNF $\alpha$-converting enzyme, which releases the soluble form of TNF $\alpha$ (sTNF $\alpha$ ). To perform their biological function, the tmTNF and sTNF monomers must aggregate to form homotrimers. Both tmTNF $\alpha$ and sTNF $\alpha$ can bind to both TNFR1 and TNFR2, although sTNF $\alpha$ demonstrates greater affinity for TNFR1 than TNFR2, which leads to inflammatory responses and cell apoptosis. Conversely, tmTNF $\alpha$ acts mainly on TNFR2, which leads to cell activation and cell survival. The isolated expression of tmTNF in transgenic mice can suppress initiation and progression of experimental autoimmune encephalitis, while maintaining the properties of autotolerance and resistance to infection. Thus, selective inhibition of sTNF/ TNFR1 signaling might be used as a therapeutic strategy for prevention of relapsing multiple sclerosis.

The increased use of anti-TNF $\alpha$ agents over the last decade has revealed a variety of immune-mediated adverse events [8]. Clinical signs of autoimmune diseases, such as urticaria, psoriasis, lupus-like syndrome, diabetes mellitus type I, and others, have been reported. In addition, numerous reports and case series of neurological adverse events due to anti$\mathrm{TNF} \alpha$ antagonists have been reported. These include demyelinating conditions, optic neuritis, chronic inflammatory demyelinating polyneuropathy, mononeuritis multiplex, GuillainBarré syndrome and others.

Several theories have been proposed to explain a potential biological relationship between TNF $\alpha$ antagonists and demyelinating disease: (a) TNF $\alpha$ blockers do not penetrate the blood-brain barrier, but enhance disease activity via an increase in peripheral T-cell autoreactive cells, which can penetrate into the central nervous system [5, 6]; (b) downregulation of TNFR2 is necessary for proliferation of oligodendrocytes and for damage repair [7-13]; (c) downregulation of the downstream production of cytokines, such as interleukin (IL)-10, and upregulation of IL-12 and interferon- $\gamma$ are associated with the demyelinating disease process [14], and (d) TNF $\alpha$ antagonists can unmask a latent infection that is critical to inciting an autoimmune demyelinating process [14].

In the literature, two types of optic neuritis have been described during treatment with infliximab: retrobulbar demyelinating optic neuritis, and bilateral anterior optic neuropathy 


\section{Case Reports in Ophthalmology}

Case Rep Ophthalmol 2016;7:345-353

(c) 2016 The Author(s). Published by S. Karger AG, Basel www.karger.com/cop

Bruè et al.: Demyelinizing Neurological Disease after Treatment with Tumor Necrosis Factor- $\alpha$ Antagonists

[15]. Furthermore, Tran et al. [15] reported on five cases of optic neuropathy associated with infliximab: one as retrobulbar optic neuropathy without pain with loss of central visual acuity and hyperreflectivity on MRI; two as bilateral optic neuropathy without pain with loss of central visual acuity and normal MRI; one as painful optic neuropathy with loss of central visual acuity (although MRI was not performed), and one as optic neuropathy with progressive central scotoma that evolved to optic atrophy and normal MRI. Optic neuropathy provoked by the use of infliximab can also be anterior or posterior.

Some studies have suggested that anti-TNF $\alpha$ agents can directly alter autoimmune responses through an autoimmune activity that is responsible for a demyelinating action. The second type of optic neuropathy associated to infliximab is a bilateral edematous anterior optic neuropathy which probably arises due to toxicity. This was described for three patients aged between 54 and 62 years, where the neuropathy occurred after the third perfusion, thus suggesting a toxic action caused by the cumulating dose. A deficit of the central or paracentral visual field evokes toxic effects rather than ischemic effects for this anterior optic neuropathy [15]. Our case is a retrobulbar optic neuropathy that was responsive to the suspension of adalimumab treatment and to the application of systemic corticosteroids.

It has been suggested that the use of TNF $\alpha$ antagonists can increase the risk of demyelinating diseases in patients with rheumatoid arthritis by about 30\% [14]; however, these data are not supported by other studies [14]. The overall prevalence of rheumatoid arthritis and multiple sclerosis in patients under TNF $\alpha$ antagonist treatment is 0.6 and $0.05 \%$, respectively [5]. The occurrence of both diseases in the same patient has also been reported [14]. This 'coincidence' should not, however, be surprising, as these two diseases share similar pathogenetic and genetic similarities [14]. In this context, patients with one autoimmune disease are at increased risk of developing another.

This paradoxical failure of anti-TNF agents in multiple sclerosis and their precipitation of demyelinating events have been explained according to several mechanisms. These have included the heterogeneity of the role and mechanism of action of TNF $\alpha$ in different autoimmune diseases, the inability of these agents to pass through the blood-brain barrier to neutralize and prevent local TNF $\alpha$-mediated tissue damage, and their as yet unidentified role in facilitating particular immune responses that contribute to demyelination.

However, there is also the question whether this association actually represents an unmasking of preexisting, presymptomatic multiple sclerosis or the onset of new demyelinating disease. It has been reported that the duration from the introduction of anti-TNF $\alpha$ antagonists to the onset of demyelinating disorders is 5 months on average. The peak age for the onset of multiple sclerosis is between 20 and 40 years, and that of rheumatoid arthritis is between 40 and 60 years. In patients who are candidates for anti-TNF $\alpha$ therapy, detailed clinical and neurological examinations are necessary.

To the best of our knowledge, we provide here the first description of Crohn's diseaseassociated optic neuropathy treated with oral steroids, where we obtained good visual outcomes. Corticosteroids have immunosuppression actions at both genomic and nongenomic levels, with effects on blood-brain barrier integrity and leukocyte chemotaxis that produce immunoglobulin, cytokine and macrophage activation. The Optic Neuritis Treatment Trial (ONTT), a multicenter prospective, randomized, placebo-controlled clinical study, evaluated the efficacy of treatment of acute optic neuritis with oral and intravenous steroids. The ONTT showed that 3 days of high-dose intravenous methylprednisolone did not change overall outcomes of visual acuity after 6 months, but did accelerate visual recovery after optic neuritis [16]. Low-dose oral steroids increased the incidence of recurrent optic neuritis for reasons that remain unclear. Treatment with steroids was found to be safe in the ONTT, 


\section{Case Reports in Ophthalmology}

Case Rep Ophthalmol 2016;7:345-353

(c) 2016 The Author(s). Published by S. Karger AG, Basel www.karger.com/cop

Bruè et al.: Demyelinizing Neurological Disease after Treatment with Tumor Necrosis Factor- $\alpha$ Antagonists

except for one patient who had acute pancreatitis and another who had acute transient depression. The debate is still ongoing on the treatment of idiopathic demyelinating optic neuritis, as intravenous steroids accelerate visual recovery and potentially postpone the insurgence of multiple sclerosis, but have not been efficacious for long-term visual function. Furthermore, in the ONTT, the contrast sensitivity, visual fields, and color vision showed persistent improvements over placebo after 6 months in the intravenous methylprednisolone group [16]. This might be explained on the basis that earlier steroid treatment is important to get functional improvements with relative preservation of retinal ganglion cells. In the present study, we recommended the suspension of adalimumab and high-dose oral steroids 1 week after the onset of the visual symptoms. This prompt treatment resulted in excellent outcomes. This was also confirmed by the ONTT, which established that administration of oral or intravenous steroids has to be within 8 days of symptom onset [16]. Indeed, treatment of hyperacute optic neuropathy with steroids prior to the onset of symptoms has been shown to provide excellent outcomes, without the patients experiencing loss of vision [17]. In summary, for acute demyelinating optic neuropathy, the two main first-line therapeutic options are to give at least 3 days of high-dose steroids as early as possible.

Patients with autoimmune optic neuritis have different pathophysiology and prognosis of optic neuropathy. A review in 2006 described patients with optic neuropathy from vasculitis (i.e., microscopic polyarteritis, Wegener's granulomatosis, Henoch-Schönlein purpura) and collagen vascular disease (i.e., systemic lupus erythematosus, Sjögren's syndrome), where steroid therapy with and without immunosuppressive therapy was given [18]. Optic neuropathy associated with Wegener's granulomatosis, where the optic nerve can be affected by contiguous orbital spread of sinonasal disease or by occlusive vasculitis, often has an unfavorable prognosis, although some patients show positive responses to steroids. With sarcoidosis, which can frequently be refractory to steroids, these cases require treatment with immunosuppressive agents (e.g., azathioprine, cyclosporine) and antimetabolites (e.g., cyclophosphamide, chlorambucil, methotrexate). However, infliximab has shown promise in the treatment of systemic sarcoidosis, although attention needs to be paid for the routine use of anti-TNF $\alpha$ agents in the treatment of inflammatory conditions with neurological manifestations, as they have been associated with demyelinating attacks [19], as seen in the present case.

Crohn's disease is an inflammatory condition affecting the colon and small intestine. Ocular inflammation has been described by Crohn's disease with a prevalence from 4 to $30 \%$, with the most common ophthalmic manifestations being uveitis, episcleritis, and scleritis. With our case here, we believe this to be the first description of Crohn's disease associated with optic neuritis where the patient responded well to high-dose oral steroids. This report thus supplies further information concerning the correct management of this complication from TNF $\alpha$ antagonists, with good visual prognosis obtained.

Finally, close follow-up and appropriate monitoring are essential. Furthermore, when these patients develop symptoms or signs of neurological adverse events, the use of TNF $\alpha$ antagonists should be discontinued, and appropriate tests should be performed. Here, oral corticosteroids appeared to be effective for this patient with Crohn's disease in the treatment of their optic neuritis due to use of the TNF $\alpha$ antagonist adalimumab.

\section{Statement of Ethics}

The authors have no ethical conflicts to disclose. 


\section{Case Reports in Ophthalmology} www.karger.com/cop

Bruè et al.: Demyelinizing Neurological Disease after Treatment with Tumor Necrosis Factor- $\alpha$ Antagonists

\section{Disclosure Statement}

The authors have no financial interests in this report.

\section{References}

1 Khanna D, McMahon M, Furst DE: Safety of tumour necrosis factor- $\alpha$ antagonists. Drug Saf 2004;27:307-324.

2 Mohan N, Edwards ET, Cupps TR, Oliverio PJ, Sandberg G, Crayton H, et al: Demyelination occurring during anti-tumor necrosis factor- $\alpha$ therapy for inflammatory arthritides. Arthritis Rheum 2001;44:2862-2869.

-3 Thomas CW Jr, Weinshenker BG, Sandborn WJ: Demyelination during anti-tumor necrosis factor- $\alpha$ therapy with infliximab for Crohn's disease. Inflamm Bowel Dis 2004;10:28-31.

-4 Shin IS, Baer AN, Kwon HJ, Papadopoulos EJ, Siegel JN: Guillain-Barré and Miller Fisher syndromes occurring with tumor necrosis factor- $\alpha$ antagonist therapy. Arthritis Rheum 2006;54:1429-1434.

5 Magnano MD, Robinson WH, Genovese MC: Demyelination and inhibition of tumor necrosis factor (TNF). Clin Exp Rheumatol 2004;22:S134-S140.

-6 Robinson WH, Genovese MC, Moreland LW: Demyelinating and neurologic events reported in association with tumor necrosis factor- $\alpha$ antagonism: by what mechanisms could tumor necrosis factor- $\alpha$ antagonists improve rheumatoid arthritis but exacerbate multiple sclerosis? Arthritis Rheum 2001;44:1977-1983.

-7 Cisternas M, Gutiérrez M, Jacobelli S: Successful rechallenge with anti-tumor necrosis factor- $\alpha$ for psoriatic arthritis after development of demyelinating nervous system disease during initial treatment: comment on the article by Mohan et al. Arthritis Rheum 2002;46:3107-3108.

-8 Titelbaum DS, Degenhardt A, Kinkel RP: Anti-tumor necrosis factor- $\alpha$-associated multiple sclerosis. Am J Neuroradiol 2005;26:1548-1550.

-9 Ruiz-Jimeno T, Carvajal A, Mata C, Aurrecoechea E: Demyelinating disease in a patient with psoriatic arthritis and family history of multiple sclerosis treated with infliximab. J Rheumatol 2006;33:14571458.

10 Dubcenco E, Ottaway CA, Chen DL, Baker JP: Neurological symptoms suggestive of demyelination in Crohn's disease after infliximab therapy. Eur J Gastroenterol Hepatol 2006;18:565-566.

11 Toussirot E, Pertuiset E, Martin A, Melac-Ducamp S, Alcalay M, Grardel B, Seror P, Perdriger A, Wendling D, Mulleman D, Beraneck L, Mariette X; Club Rheumatismes et Inflammation: Association of rheumatoid arthritis with multiple sclerosis: report of 14 cases and discussion of its significance. J Rheumatol 2006;33:1027-1028.

-12 Noseworthy JH, Lucchinetti C, Rodriguez M, Weinshenker BG: Multiple sclerosis. N Engl J Med 2000;343:938-952.

-13 van Boxel-Dezaire AH, Hoff SC, van Oosten BW, Verweij CL, Dräger AM, Adèr HJ, van Houwelingen JC, Barkhof F, Polman CH, Nagelkerken L: Decreased interleukin-10 and increased interleukin-12p40 mRNA are associated with disease activity and characterize different disease stages in multiple sclerosis. Ann Neurol 1999;45:695-703.

14 Bernatsky S, Renoux C, Suissa S: Demyelinating events in rheumatoid arthritis after drug exposures. Ann Rheum Dis 2010;69:1691-1693.

15 Tran THC, Milea D, Cassoux N, Bodaghi B, Bourgeois P, LeHoang P: Névrite optique rétrobulbaire associée au traitement par infliximab. J Fr Ophtalmol 2005;28:201-204.

16 Beck RW, Gal RL: Treatment of acute optic neuritis: a summary of findings from the optic neuritis treatment trial. Arch Ophthalmol 2008;126:994-995.

17 Plant GT, Sibtain NA, Thomas D: Hyperacute corticosteroid treatment of optic neuritis at the onset of pain may prevent visual loss: a case series. Mult Scler Int 2011;2011:815068.

-18 Goodwin J: Autoimmune optic neuropathy. Curr Neurol Neurosci Rep 2006;6:396-402.

19 Pula JH, Macdonald CJ: Current options for the treatment of optic neuritis. Clin Ophthalmol 2012;6:1211-1223. 


\section{Case Reports in Ophthalmology}

(C) 2016 The Author(s). Published by S. Karger AG, Basel www.karger.com/cop

Bruè et al.: Demyelinizing Neurological Disease after Treatment with Tumor Necrosis Factor- $\alpha$ Antagonists
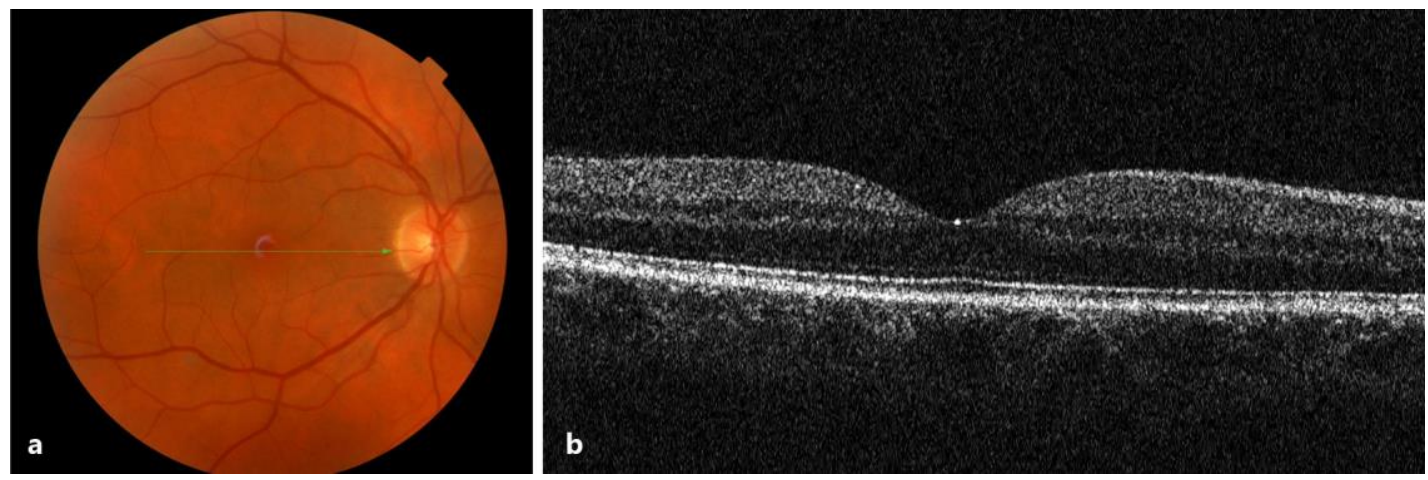

Fig. 1. Ophthalmoscopy of the right eye shows a normal macula (a). SD-OCT shows a normal foveal profile and a preserved retinal structure (b). 


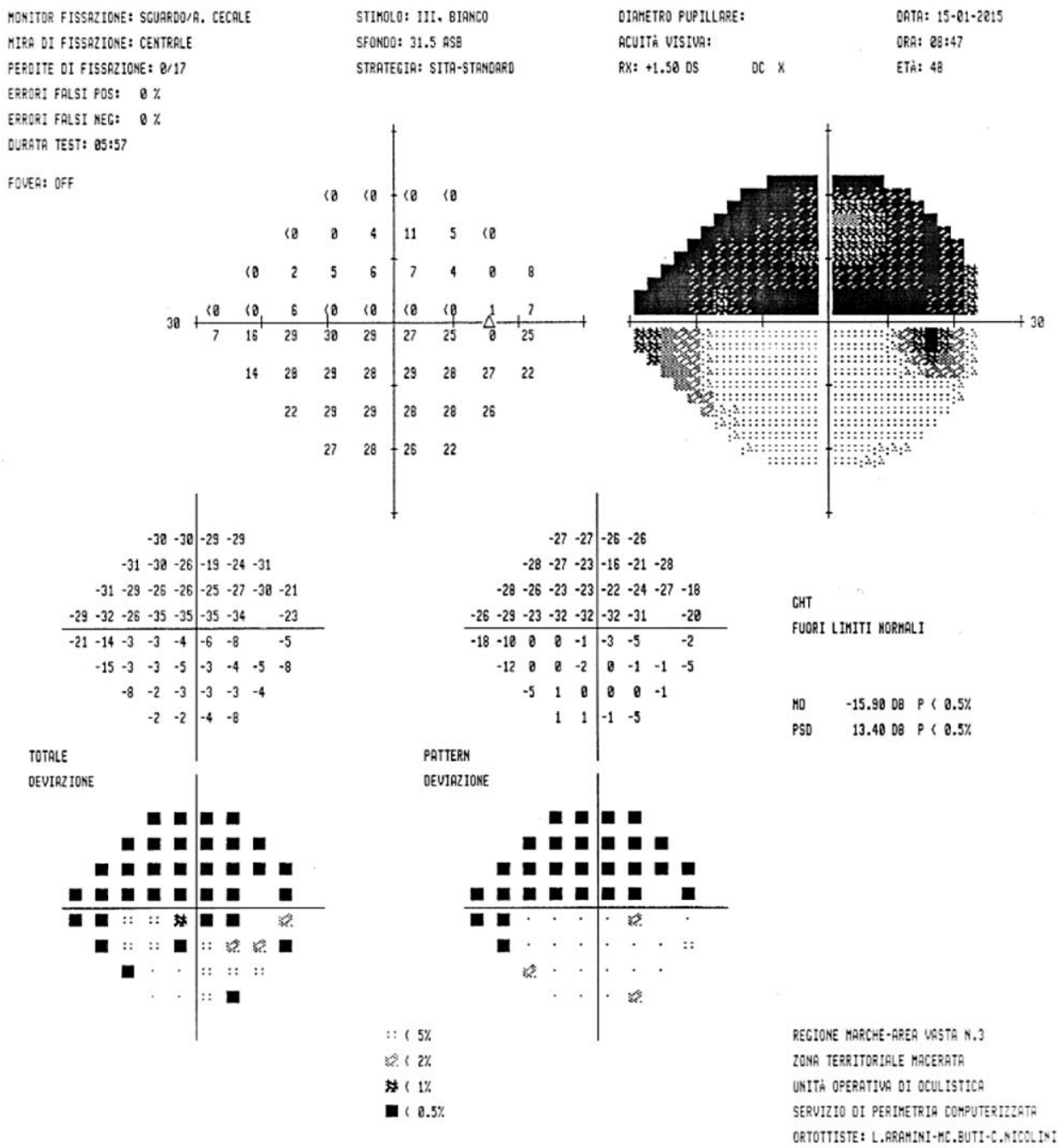

Fig. 2. Visual field examination demonstrated a superior hemianopsia in the right eye. 

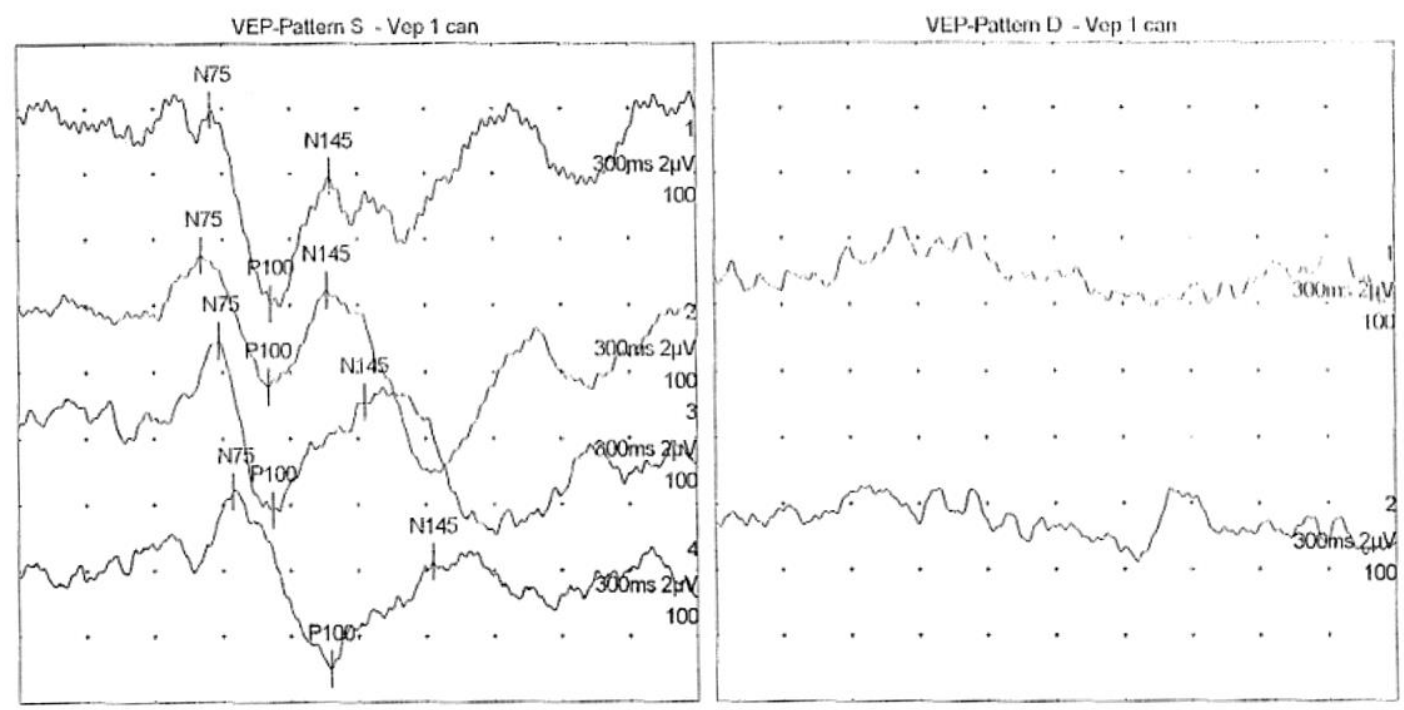

Fig. 3. The patient's VEPs were reduced, with prolonged latency, compatible with an optic neuritis diagnosis. 